$17^{\text {th }}$ International Congress of Metrology, 14007 (2015)

DOI: $10.1051 /$ metrology $/ 201514007$

(C) Owned by the authors, published by EDP Sciences, 2015

\title{
Estimation of the measurement uncertainty of LNE's metrological Atomic Force Microscope using virtual instrument modeling and Monte Carlo Method
}

\author{
Paul Ceria ${ }^{1, a}$, Sebastien Ducourtieux ${ }^{1}$ and Younes Boukellal ${ }^{1}$ \\ ${ }^{1}$ Laboratoire National de metrologie et d'Essais - 29 avenue Roger Hennequin, 78197 Trappes - France
}

\begin{abstract}
Résumé. Un Microscope à Force Atomique métrologique (mAFM) est développé au Laboratoire National de métrologie et d'Essais (LNE). Le mAFM est consacré à la mesure traçable des structures à l'échelle nanométrique et à l'étalonnage d'étalons de transfert dédiés aux microscopes à champ proche et électroniques. Le déplacement relatif de l'échantillon par rapport à la pointe est de $60 \mu \mathrm{m}$ dans le plan XY et de $15 \mu \mathrm{m}$ sur l'axe Z. La traçabilité au mètre tel qu'il est défini par le Système International d'unités (SI) est assurée grâce à quatre interféromètres différentiels utilisés dans une configuration originale [1]. La conception de l'instrument est optimisée pour minimiser les principales composantes dans le bilan d'incertitude, telles que: les dilatations thermiques, les erreurs d'Abbe, les erreurs géométriques [1]. L'incertitude attendue pour la mesure de position de la pointe par rapport à l'échantillon est d'environ $1 \mathrm{~nm}$ pour la gamme entière de déplacement, sans tenir compte de la contribution de l'interaction pointe/échantillon. Un premier bilan d'incertitude a été réalisé grâce à des évaluations expérimentales des principales sources d'erreur qui perturbent le processus de mesure [1-2]. Pour les autres composantes, leur évaluation est complexe ou expérimentalement impossible. Pour affiner le bilan d'incertitude, un modèle numérique de l'instrument (AFM virtuel) a été développé. Le modèle permet d'évaluer tous les composants (mécaniques et optiques) liés à la géométrie du système de mesure de position et permet également de prendre en compte les composantes évaluées expérimentalement et enfin de déterminer l'incertitude de mesure en utilisant la méthode de Monte-Carlo [3-4].
\end{abstract}

\section{Introduction}

Since their invention in 1986 [5], Atomic Force Microscope (AFM) has become an essential tool to characterize structures at the nanometre scale. To improve the accuracy of dimensional measurements carried out with an AFM, users need to calibrate their instruments by using transfer standards usually materialized by $1 \mathrm{D}$ or $2 \mathrm{D}$ gratings [6] whose pitch and step height values are measured and recorded in a calibration certificate and associated to the measurement uncertainty. To get these reference values, the standards are measured by a reference instrument as for example a metrological Atomic Force Microscope (mAFM). Such an instrument has been developed at LNE as in other National Metrology Institutes [7-8]. A full description of this mAFM is given in [1]. It's a short range metrological AFM (figure 1) whose measuring volume is $60 \times 60 \times 15 \mu \mathrm{m}^{3}$.

To measure the position of the sample relative to the tip and to obtain a measurement directly traceable to the $\mathrm{SI}$, the mAFM uses interferometers whose He-Ne laser sources are frequency-calibrated. The interferometric

a Email de correspondance de l'auteur : paul.ceria@lne.fr position measurement system is composed of four interferometers whose measurement axes have been tilted with respect to $\mathrm{x}, \mathrm{y}$ and $\mathrm{z}$ axis in order to place all the interferometers in a more favourable symmetric configuration below the sample and the scanning stage. Each interferometer is located on one corner of a square pyramid (figure 2). Interferometers are placed in a horizontal plane below the sample, symmetrically distributed around the tip which is centred at the origin of XYZ coordinate system. The beams are tilted of $35^{\circ}$ from the $\mathrm{XY}$ plane and rotated at $45^{\circ}$ around $\mathrm{z}$ direction.

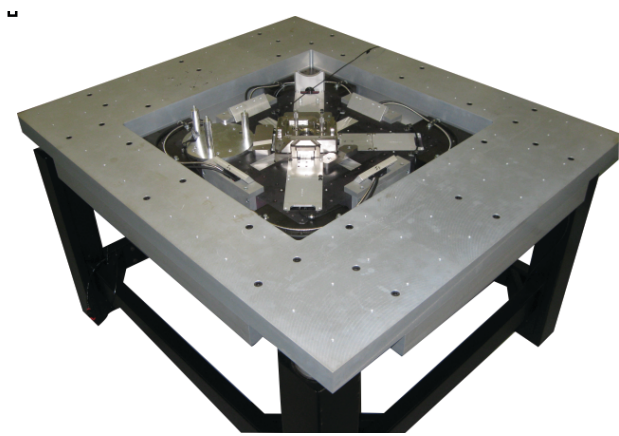

Figure 1. Photo of the LNE's mAFM. 


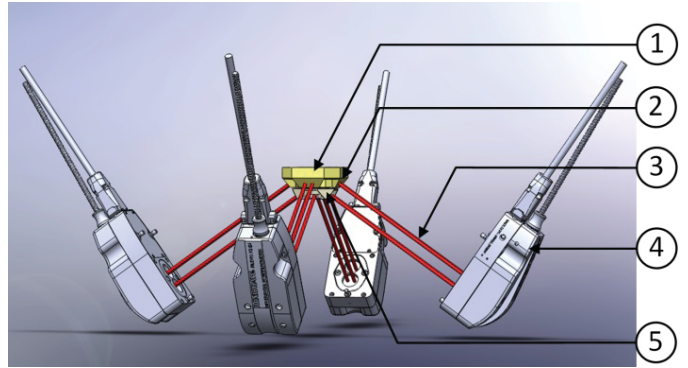

Figure 2. The interferometric position measurement system with (1) the reference prism, (2) a mirror face, (3) the interferometer beams, (4) a differential double path interferometer, (5) the measurement prism.

To measure the displacement, the interferometers point at mirrors directly polished on two prisms made of Zerodur (low thermal expansion ceramic). Due to the geometry of the system, the two prisms have the shape of a truncated inverted four-face pyramid with a half top angle of $55^{\circ}$ (figure 3 ).

The first prism is linked to the AFM head and supports the reference mirrors for the interferometers. It is associated with the tip position. The second one, the measurement prism, supports the mirrors associated with the sample displacement. To reduce dead path errors, the two prisms are positioned and sized to make the mirror faces coplanar.

\section{The modelling process}

In this configuration, $\mathrm{XYZ}$ positions are obtained by linear combinations of each interferometer $\left(\mathrm{I}_{1}, \mathrm{I}_{2}, \mathrm{I}_{3}, \mathrm{I}_{4}\right)$ according to the following equations:

$$
\left\{\begin{array}{c}
X=\frac{I_{1}-I_{2}-I_{3}+I_{4}}{4 \cdot \cos 45 \cdot \cos 35} \\
Y=\frac{-I_{1}-I_{2}+I_{3}+I_{4}}{4 \cdot \sin 45 \cdot \cos 35} \\
Z=\frac{-I_{1}-I_{2}-I_{3}-I_{4}}{4 \cdot \sin 35}
\end{array}\right.
$$

With such a configuration, the evaluation of certain contributions in the uncertainty budget is experimentally difficult if not impossible. To precisely determine their impact on the MAFM, a numerical model of the MAFM has been developed. It represents a faithful modelling of the metrological chain. It has been developed under Matlab using object oriented programming. This model takes into account the laser beams, the AFM's tip position, the measurement and reference prisms and their mirrors as shown on figure 3. Displacements in six degrees of freedom can be generated on the mobile prism (sample translation) or any object to evaluate measurement errors.

\subsection{The prisms modelling}

The mirrors are modelled using a 3D cloud of points which will allow the easy transfer of experimental data to the model in order to take into account the real shape of the mirrors (shape, roughness for instance). The model can control the length and height of the mirror, and also the number of points inside the cloud. The mirror positions are saved in a matrix containing three vectors (XYZ) and a fourth to facilitate the calculations using a dedicated homogeneous formalism. This formalism is used to rotate and translate the mirror in space. The number of points in the cloud can be adapted to fit the experimentally measured data for shape and roughness. The mirror shapes can also be controlled using analytic functions. The mirror configuration respects the one of the real mAFM's prisms.

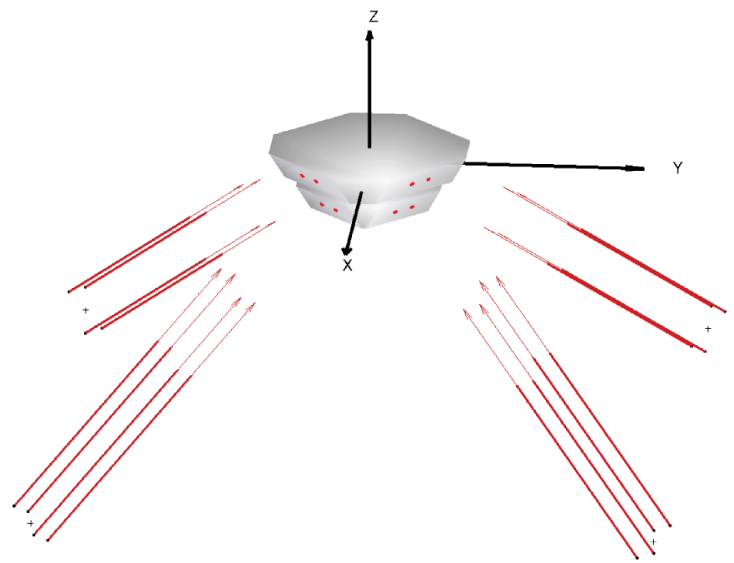

Figure 3. Matlab view of the interferometer position measurement system model taking into account the four interferometers, their sixteen Gaussian beams, and the two prisms with the eight mirrors.

\subsection{The interferometer beam modelling}

The interferometers are not modelled in our first approach. Only the four beams emerging from the differential double pass interferometer are taken into account. These beams are modelled with a source point, a direction defined with a vector targeting the mirror and a Gaussian profile. In this way, the four beams act as four mechanical probes that only measure the distance from the source to the corresponding mirror. It takes into account the Gaussian profile and the impacted surface of the mirror as shown in the figure 4. To feed the model with experimental data, the real interferometer beams have been analysed to get their shapes and dimensions. The data are computed with Matlab to found the best coefficients that define the modelled Gaussian profile (standard deviation, mean and amplitude). For each beam, the distances are calculated and then processed to evaluate the differential displacement between the reference mirror and the mobile mirror as measured by one interferometer $\left(\mathrm{I}_{1}, \mathrm{I}_{2}, \mathrm{I}_{3}\right.$ or $\left.\mathrm{I}_{4}\right)$. 


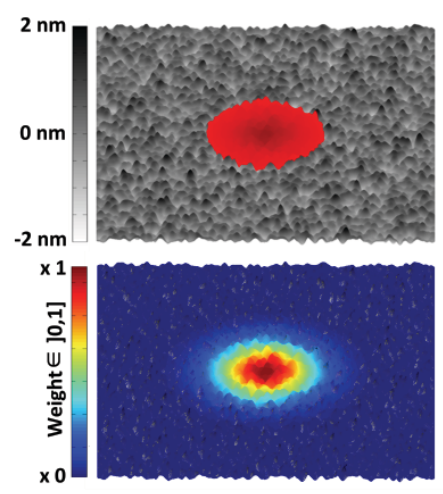

Figure 4. Representation of the Gaussian beam impacting a rough mirror as modelled under Matlab.

\section{Monte-Carlo method}

With this model, it is possible to simulate complex displacements of the prisms taking into account the measurement errors. Therefore, different sources of uncertainty can be identified, such as: orthogonality error of the mirrors, misalignments of the interferometer beams, parasitic rotations of the scanning stage, Abbe errors, roughness and shapes errors of the mirrors, basic linear dilatation of the system, positioning error of the measurement prism. To obtain a probability density function (PDF) of the measurement error results, the above described simulation process is executed many times, with different influencing parameters generated randomly by the Monte-Carlo Method (MCM). The measurement uncertainty can be derived from the calculated density distribution. Furthermore, the number of simulations in the MCM should be chosen large enough to ensure that the result quality is sufficiently high. The probability density function output can be obtained through fitting the simulation results and used to calculate the standard deviation defining the measurement uncertainty. The simulation is running on a cluster with 32 cores to reduce calculation times.

The figure 5 shows the position error processing. First, the model creates the prisms and the interferometers without default, that means, the prisms and the interferometer beams are perfectly geometric and aligned. Then, the user chooses several parameters to set up the MCM tool, as for example the tolerance number for an unique component default. For the moment in the presented poster, the uncertainty measurement results are independents for each component. User can also select a law distribution for the component default. The MCM allows processing a wide range of PDF's laws, and $10^{5}$ random draws.

\section{Measurement uncertainty}

This model allows an estimation of several components that perturb the measurement process by using Monte-Carlo method. This model simulates errors on any object and give the impact of the error on the measurement uncertainty. The table 1 gives the different components that the model can simulate.

After the measurement process, the model recovers the output PDF. Most of the time, the PDF is a Gaussian distribution. Then the distribution is fitted, and the standard deviation is determined. Therefore, the uncertainty budget is evaluated for each component.

Table 1. List of the model parameters that can be adjusted to modify the geometry of the measurement system.

\begin{tabular}{cl} 
Object & Simulated components \\
\hline \multirow{3}{*}{ Mirror } & Orthogonality \\
& Shape \\
& Roughness \\
\hline \multirow{3}{*}{ Prism } & Sample displacement \\
& Prism shape \\
& Dilatation \\
& Parasitic rotations \\
& Positioning errors \\
& Thickness of the sample \\
\hline \multirow{3}{*}{ Beam } & Distance measurement \\
& Beam radius \\
& Beam orientation \\
& Beam misalign \\
& Cosine error \\
\hline \multirow{3}{*}{ interferometer } & Differential measurement \\
& Orientation \\
& Misalignment \\
& Chassis dilation \\
& Abbe errors
\end{tabular}

\section{Conclusion}

Currently, the model shows that orthogonality and Abbe error are the most important contributions in the measurement uncertainty. We have to improve our result with more experimental data to refine some large tolerance we are currently using in the model. In the next few months, the model will be completed with the geometrical calibration data of the prism.

The final work will allow evaluating the contribution of a much more components that could perturb the measurement process, to determine the interaction between them and their sensitivities and finally to establish the uncertainty budget for the mAFM. Thanks to the object oriented programming, the model is easily reconfigurable to other instruments geometry to evaluate the measurement uncertainties and could be applied on other measurement machines/instruments.

\section{Acknowledgments}

The research was supported by EURAMET joint research project "Six degrees of freedom" receiving funding from the European Community's Seventh Framework Programme, ERA-NET Plus, under Grant Agreement No. 217257. 


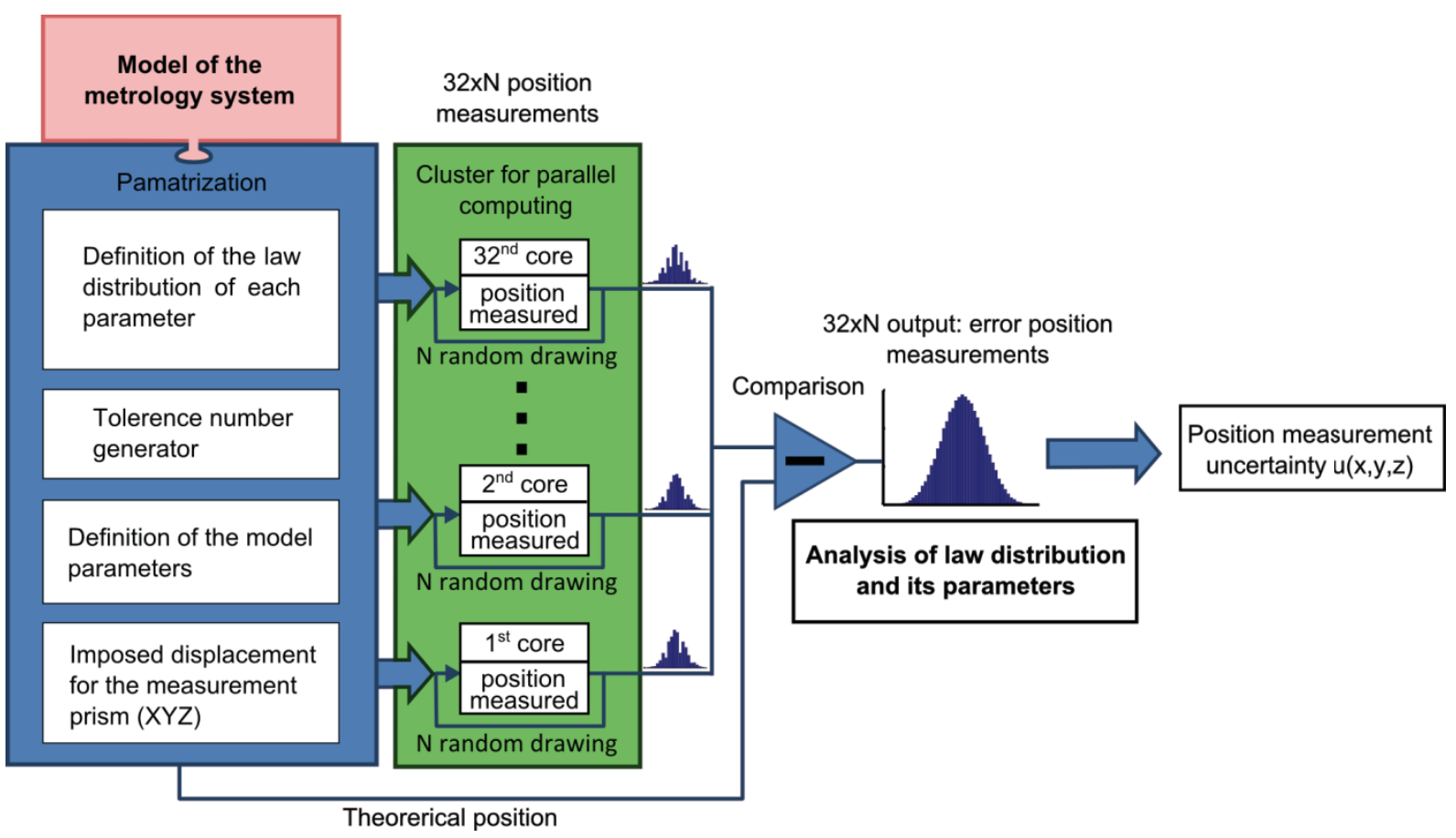

Figure 5. Model diagram method

\section{References}

1. S. Ducourtieux, B. Poyet, Development of a metrological atomic force microscope with minimized Abbe error and differential interferometer-based real-time position control, Measurement Science and Technology 22(9):094010 (2011)

2. Y. Boukellal, S. Ducourtieux, B. Poyet, Improvement of the LNE's metrological Atomic Force Microscope (mAFM) performance: Design of new mAFM head dedicated for nanometrology applications, 16th International Congress of Metrology 06008 (2013)

3. JCGM-WG1 101, Evaluation of measurement data Supplement 1 to the "Guide to the expression of uncertainty in measurement" - Propagation of distributions using a Monte Carlo method, ISO/IEC Guide 98-3-1 (2008)

4. JCGM 100, Evaluation of measurement data - Guide to the expression of uncertainty in measurement, ISO/IEC Guide 98-3 (2008)

5. G. Binnig, C. F. Quate, C. Gerber, Atomic Force Microscope, Phys. Rev. Lett. 56(9):930-3

6. Surface chemical analysis - Scanning-probe microscopy Determination of geometric quantities using SPM: Calibration of measuring systems. ISO 11952:2014

7. F. Meli, R. Thalmann, Long-range AFM profiler used for accurate pitch measurements, Meas Sci Technol 9(7):1087 (1998)

8. H. U. Danzebrink, L. Koenders, G. Wilkening, A. Yacoot, H. Kunzmann, Advances in Scanning Force Microscopy for Dimensional Metrology, CIRP Ann. Manuf. Technol. 55(2):841-78 (2006) 